\title{
Efficacy of an Arthroscopic Virtual Based Simulator for Orthopedic Surgery Residents by Year in Training
}

\author{
Shahram Yari, MD1, Chanakya Jandhyala, MD1, Behnam Sharareh, MD1, Aravind Athiviraham, MD², Theodore B. Shybut, \\ MD'
}

${ }^{1}$ Baylor College of Medicine, Houston, TX, USA, ${ }^{2}$ University of Chicago, Chicago, IL, USA.

Objectives: Arthroscopic virtual-reality stimulators are an attractive option for resident training and are increasing across training programs. However, no study has analyzed the utility of the simulator for trainees based on their post-graduate level of training (PGY-level). The primary aim of this study was to determine the utility of the ArthroS ${ }^{\mathrm{TM}}$ arthroscopic simulator for orthopedic trainees based on their level of training (to determine at what point in training the simulator offers the most benefit for trainees). We hypothesized that all levels would show similar improvements in performance following completion of the training modules.

Methods: Eighteen orthopedic surgery residents performed pre-training diagnostic knee and shoulder tasks on the ArthoS ${ }^{\mathrm{TM}}$ simulator. Subjects completed a series of training modules, then repeated the diagnostic tasks. Mean composite scores were calculated. Group differences for discrete variables were evaluated using ANOVA (analysis of variance). Correlation coefficients $\left(R^{2}\right)$ were calculated for improvements in mean composite score as a function of subject year-in-training.

Results: Average improvement in composite score for participants as a whole was 11.2 points [SD 10; $p$-value 0.0003] for the knee simulator and 14.9 points [SD 10.9; $p$-value 0.0352 ] for the shoulder simulator. When broken down by PGY-level, all groups showed improvement, with greater improvements seen for junior-level residents in the knee simulator and greater improvements seen for senior-level residents in the shoulder simulator. ANOVA for the score improvement variable in the knee simulator data amongst the different PGY-groups yielded an $\mathrm{f}$-value of 1.640 ( $p$-value 0.2258) and that for the shoulder simulator data amongst the different PGY-groups yielded an $f-$ value of 0.2292 ( $p$-value 0.917 ). The correlation coefficient $\left(r^{2}\right)$ was -0.866 for the knee score improvement and 0.887 for the shoulder score improvement (Image 1).

Conclusion: We found that residents training on a virtual arthroscopic simulator made significant improvements in both knee and shoulder arthroscopic skills. Most importantly, we report for the first time that the knee simulator appears to be more beneficial for junior residents, while the shoulder simulator appears to be more beneficial for senior residents. Our study adds to the mounting evidence supporting virtual arthroscopic simulator-based training for orthopedic surgery residents. 

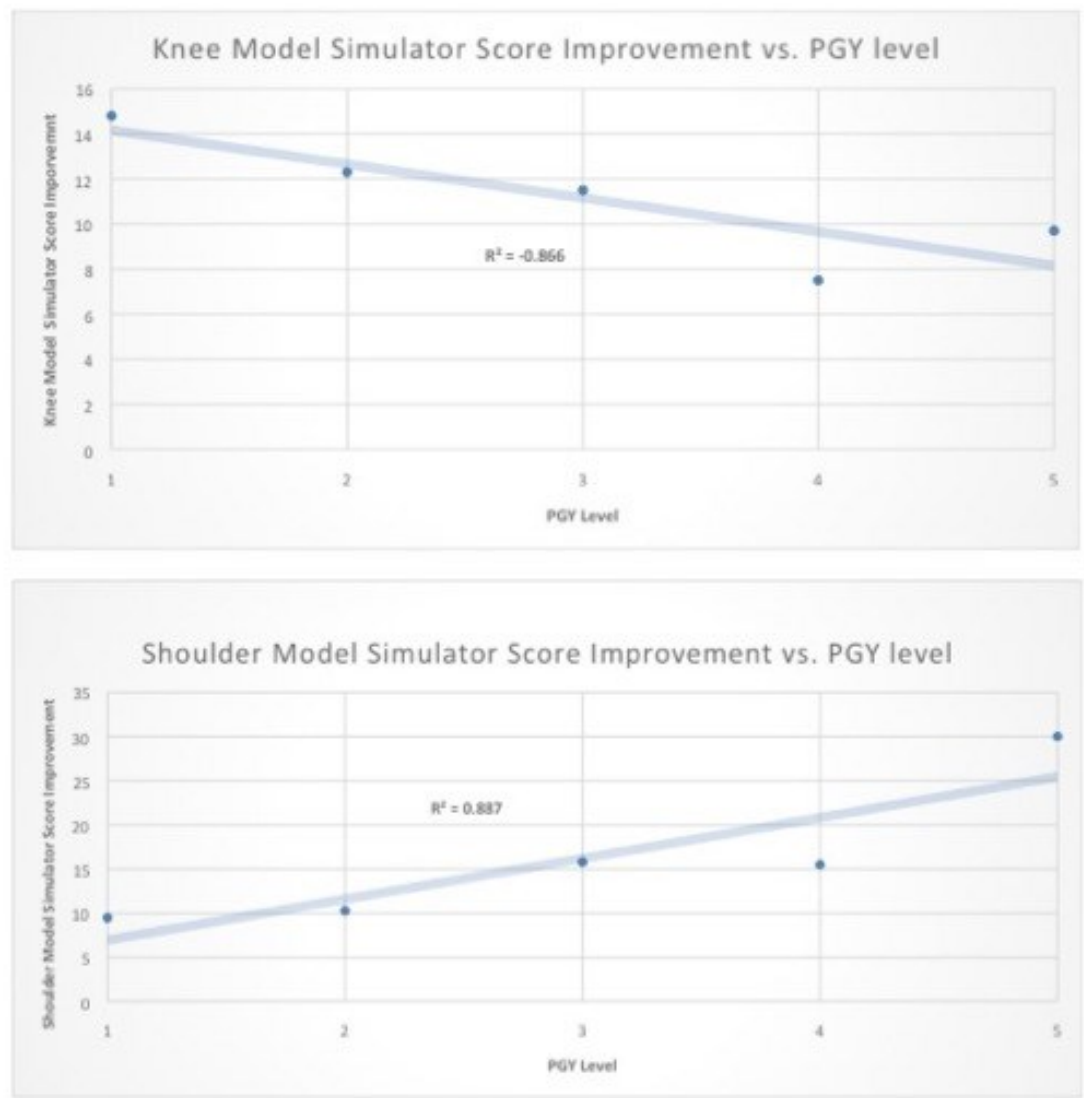

The Orthopaedic Journal of Sports Medicine, 6(7)(suppl 4)

DOI: $10.1177 / 2325967118 S 00130$

(CThe Author(s) 2018 